\title{
2FHL: The second Catalog of Hard Fermi-LAT Sources
}

\author{
Marco Ajello*† \\ Clemson University \\ E-mail: majello@clemson.edu
}

\author{
Alberto Domìnguez \\ Clemson University \\ E-mail: alberto@clemson.edu \\ Jamie Cohen \\ University of Maryland \\ E-mail: jcohendastro.umd.edu
}

\section{Sara Cutini}

Agenzia Spaziale Italiana (ASI) Science Data Center

E-mail: sara.cutini@asdc.asi.it

\section{Dario Gasparrini}

Agenzia Spaziale Italiana (ASI) Science Data Center

E-mail: dario.gasparrini@asdc.asi.it

The Fermi Large Area Telescope (LAT) has been routinely gathering science data since August 2008, surveying the full sky every three hours. The first Fermi-LAT catalog of sources detected above $10 \mathrm{GeV}$ (1FHL) relied on three years of data to characterize the $>10 \mathrm{GeV}$ sky. The improved acceptance and point-spread function of the new Pass 8 event reconstruction and classification together with six years of observations now available allow the detection and characterization of sources directly above $50 \mathrm{GeV}$. This closes the gap between ground-based Cherenkov telescopes, which have excellent sensitivity but small fields of view and duty cycles, and all-sky observations at $\mathrm{GeV}$ energies from space. In this contribution we will present the second catalog of hard Fermi-LAT sources detected at $>50 \mathrm{GeV}$. We will discuss the properties of the extragalactic and Galactic source populations with an emphasis on the detection of spatially extended sources in the plane of our Galaxy.

The 34th International Cosmic Ray Conference,

30 July- 6 August, 2015

The Hague, The Netherlands

\footnotetext{
* Speaker.

On behalf of the Fermi-LAT collaboration.
} 


\section{Introduction}

The Large Area Telescope (LAT) on board the Fermi satellite has been efficiently surveying the GeV sky detecting over 3000 sources in just four years of exposure (see the 3FGL catalog, (1)). These sources are detected in the $0.1-300 \mathrm{GeV}$ band and given Fermi's peak sensitivity at $\sim 1 \mathrm{GeV}$ are representative of the $\mathrm{GeV}$ sky. On the other hand, Cherenkov telescopes, with their good angular resolution and excellent point-source sensitivity have been exploring, due to their limited field of views, small patches of the $>50 \mathrm{GeV} \mathrm{sky}{ }^{1}$. In the effort to fill the gap, the LAT-collaboration released a catalog of sources detected, in 3 years, at $>10 \mathrm{GeV}$ (so called 1FHL catalog, (2)).

Recently a new event reconstruction and characterization analysis (known as Pass 8, (3)) has been developed by the Fermi-LAT collaboration. Pass 8 significantly improves the background rejection, point-spread function (PSF), effective area of the LAT and helps understanding its systematic uncertainties. All these impressive improvements lead to a significant increase of the LAT sensitivity (Atwood et al., 2013a,b). Furthermore, these improvements are specially significant at $E>50 \mathrm{GeV}$ with an increase in the acceptance of $\gtrsim 25 \%$ and an improvement in the PSF by a factor between $20 \%$ at $50 \mathrm{GeV}$ and $50 \%$ at $500 \mathrm{GeV}$. At these high energies, because of the almost lack of background, the sensitivity of Fermi-LAT improves almost linearly with time as it should in a photon-limited regime (as opposed to a background-limited regime where the sensitivity improves with the square root of exposure time).

Taking advantage of the improvements delivered by Pass 8, we are preparing an all-sky catalog of sources detected at $E>50 \mathrm{GeV}$ in 80 months of data. These sources will constitute the second catalog of hard Fermi-LAT sources (2FHL). This proceeding shows that the 2FHL catalog provides a view of the high-energy sky that is complementary to that of the 3FGL catalog and has the potential to allow for unprecedented broad band studies of the spectral energy distribution (SED) of old and newly discovered sources and to increase the efficiency of the searches of current Cherenkov telescopes.

\section{The 2FHL Catalog}

In about 80 months of exposure, Fermi-LAT has detected approximately 61000 photons (belonging to the P8 source class) all-sky at $>50 \mathrm{GeV}$. The preliminary all-sky map in Fig. 1 shows that Fermi-LAT observes large scale diffuse emission in the direction of our Galaxy and coincident with the so-called Fermi bubbles $(4 ; 5)$ as well as many point-like sources.

The analysis to detect sources is performed similarly to the other Fermi-LAT catalogs. The first step comprises the detection of source candidates (so called seeds) as fluctuations above the background. The sky is then divided into region of interests (ROIs), for which a sky model is built including all point sources in the ROI and also the Galactic and isotropic diffuse models (6). This model is fitted to the data via a standard maximum-likelihood unbinned algorithm. The fit is typically repeated twice and in between the two fits the source position is optimized using standard Fermi tools ${ }^{2}$.

\footnotetext{
${ }^{1}$ VERITAS, H.E.S.S and MAGIC have successfully lowered, in recent years, their low energy threshold and have started exploring the sub- $100 \mathrm{GeV}$ band.

${ }^{2}$ In this case gtfindsrc was used, see http://fermi.gsfc.nasa.gov/ssc/data/analysis/software.
} 
Figure 1: Adaptively smoothed count map, in Galactic coordinates, at $>50 \mathrm{GeV}$.

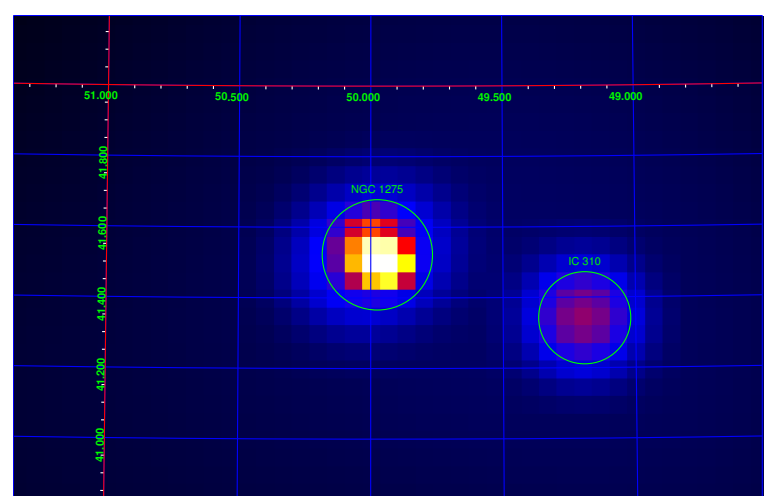

Figure 2: Adaptively smoothed count map of the region around NGC 1275 and IC 310 separated by roughly $0.6 \mathrm{deg}$.

Once a best fit has been found for a given ROI, the spectra of all sources are generated in three logarithmic energy bins from $50 \mathrm{GeV}$ to $2 \mathrm{TeV}$.

The 2FHL catalog comprises (preliminarily) 360 sources detected and characterized exclusively at $>50 \mathrm{GeV}$. For comparison, $\sim 161$ are the known Very High-energy (VHE) sources reported in the $\mathrm{TeVCat}^{3}$. The $2 \mathrm{FHL}$ thus represents a leap forward for the study and characterization of the VHE sky. It is interesting to note that 2FHL sources are selected on the basis of their average flux and thus the 2FHL catalog may be considered an unbiased census of the VHE sky. A preliminary association shows that $\sim 942 \mathrm{FHL}$ sources are detected in TeVCat as well and that the 2FHL comprises $\sim 110$ sources that were not detected in either the 1FHL or TeVCat.

Of all sources detected in the 2FHL, blazars (or blazar-like objects) represent $\sim 75 \%$, while unassociated sources and Galactic sources make up $\sim 15 \%$ and $\sim 10 \%$ respectively.

\subsection{Angular and Position Resolution}

Pass 8 improves the PSF of the LAT at all energies. Above $50 \mathrm{GeV}$ the PSF has a $68 \%$ containment radius of $\sim 0.1^{\circ}$ and remains constant with energy. Such PSF, not dissimilar from the one of Cherenkov telescopes, allows Fermi-LAT to localize sources with an average precision of $4^{\prime}$ at $95 \%$ confidence. Fig. 2 shows that Fermi-LAT can easily separate nearby sources like it is the case for NGC 1275 and IC 310. However, such resolution is most useful in the plane of the Galaxy, where it helps to solve crowded regions and resolve extended sources.

\subsection{Spectra}

The 2FHL catalog will report, for every source, 3 energy-bin spectra in the energy range $50 \mathrm{GeV}-2 \mathrm{TeV}$. These together with the spectra reported in the 3FGL and 1FHL catalogs allow us

\footnotetext{
${ }^{3}$ http://tevcat.uchicago.edu
} 


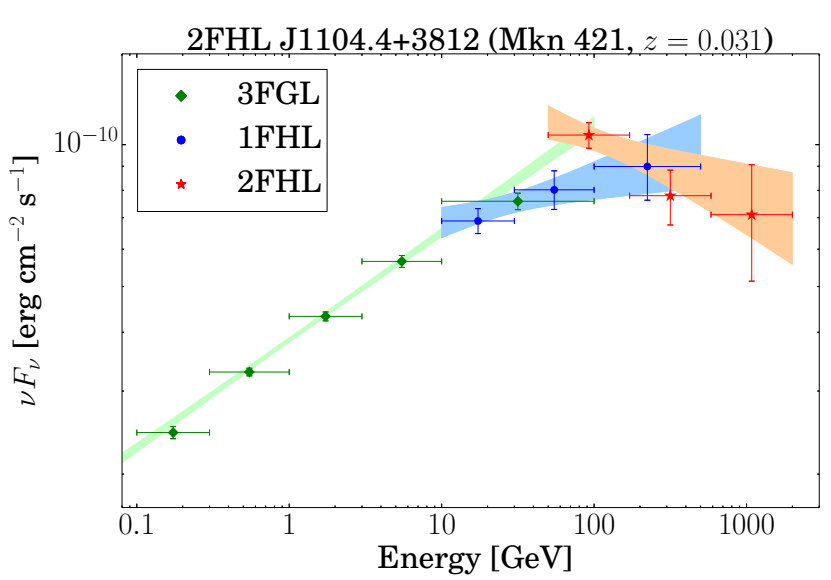

Figure 3: Preliminary spectrum of Mkn 421 in 2FHL together with data from 1FHL and 3FGL. The three catalogs rely on different exposure times.

the LAT to characterize the SEDs of sources over almost 4 decades in energy. An example is reported, for Mkn 421, in Fig. 3. High synchrotron peaked (HSP) blazars, like Mkn 421, are detected by Fermi-LAT, typically, as power-law sources with a photon index of $\sim 1.8$ (when integrated over the full energy range as in the 3FGL). It is clear that above $50 \mathrm{GeV}$ (e.g. the 2FHL) Fermi-LAT samples already the descending part of the high-energy peak of the spectral energy distribution (SED) of such sources and that the data from the 3FGL, 1FHL and 2FHL catalogs allow us to characterize the emission at the peak of such sources rather well. While Mkn 421 represents probably the best example, Fig. 4 shows that such conclusion holds, on statistical grounds, for most BL Lacs detected by Fermi-LAT.

The 2FHL catalog comprises BL Lacs detected up to redshift $\sim 2.0$. The improved reconstruction and increased acceptance allow Fermi-LAT to detect photons up to $\sim 2 \mathrm{TeV}$ (see e.g. Fig. 3). Both these aspects enable studies of the extragalactic background light (EBL) which can absorb high-energy photons emitted from sources at cosmological distances (EBL, $(7 ; 8 ; 9)$ ). BL Lacs with substantial high-energy emission at e.g. $>100 \mathrm{GeV}$ are excellent probes of the EBL and have already been used with success to constrain the $\gamma$-ray opacity of the Universe $(10 ; 11 ; 12)$. We expect that the 2FHL, thanks to improved acceptance of high-energy photons yielded by Pass 8 , will enable accurate studies of the EBL.

\section{Conclusions and Outlook}

The 2FHL catalog of Fermi-LAT sources detected at $>50 \mathrm{GeV}$ represents an unbiased census of the VHE sky. This work probes larger energies than any previous Fermi-LAT catalogs thanks to the improved Pass 8 dataset. The view of the $\gamma$-ray sky delivered by the 2FHL is complementary and different than that of the (e.g.) 3FGL catalog. Indeed, we find that most extragalactic sources are softer in the 2FHL than in the 3FGL, implying a peak of their spectral energy distribution somewhere within the Fermi band.

The 2FHL catalog will comprise sources detected on the basis on their average flux. Since $75 \%$ of the detected sources are blazars, the 2FHL will yield important information for the gener- 


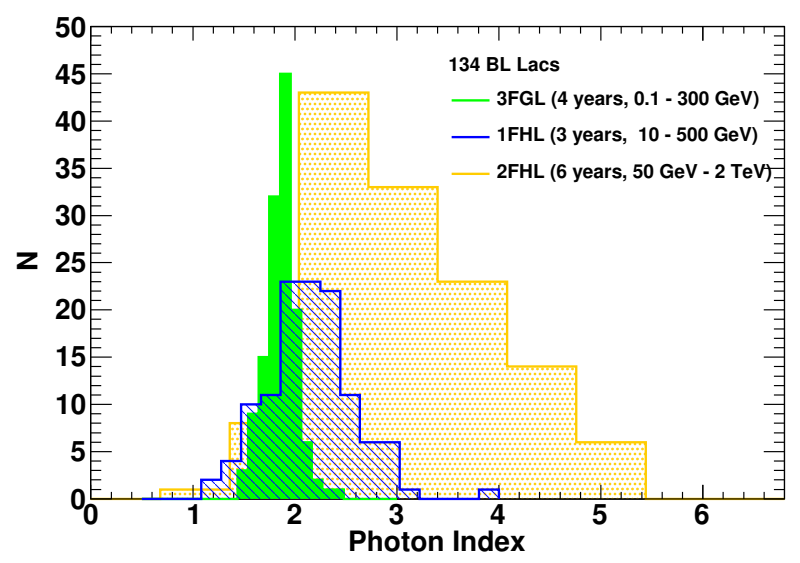

Figure 4: Distribution of the power-law photon indices for 134 BL Lacs detected in the 3FGL, 1FHL and 2FHL catalogs. Note the softening of the photon index when moving from lower energies (3FGL) to higher energies (1FHL and 2FHL).

ation of the high-energy part of the $\gamma$-ray background $(13 ; 14)$. It will also allow a first estimate of the source count distribution of VHE sources acting as a pathfinder for the surveys performed by the upcoming Cherenkov Telescope Array (15).

Finally, the good angular resolution achieved, thanks to Pass 8, by Fermi-LAT at $>50 \mathrm{GeV}$ will allow unprecedented studies of the Galaxy allowing to resolve crowded regions as well as new extended sources. We envision that this aspect of 2FHL will act as a lower energy counterpart of the H.E.S.S. Galactic plane survey (16) and the survey carried out by HAWC (17).

\section{Acknowledgments}

The Fermi LAT Collaboration acknowledges generous ongoing support from a number of agencies and institutes that have supported both the development and the operation of the LAT as well as scientific data analysis. These include the National Aeronautics and Space Administration and the Department of Energy in the United States, the Commissariat à l'Energie Atomique and the Centre National de la Recherche Scientifique / Institut National de Physique Nucléaire et de Physique des Particules in France, the Agenzia Spaziale Italiana and the Istituto Nazionale di Fisica Nucleare in Italy, the Ministry of Education, Culture, Sports, Science and Technology (MEXT), High Energy Accelerator Research Organization (KEK) and Japan Aerospace Exploration Agency (JAXA) in Japan, and the K. A. Wallenberg Foundation, the Swedish Research Council and the Swedish National Space Board in Sweden. Additional support for science analysis during the operations phase is gratefully acknowledged from the Istituto Nazionale di Astrofisica in Italy and the Centre National d'Etudes Spatiales in France.

\section{References}

[1] The Fermi-LAT Collaboration, Fermi Large Area Telescope Third Source Catalog, ArXiv:1501.02003 (Jan., 2015) [arXiv:1501.0200]. 
[2] M. Ackermann, M. Ajello, A. Allafort, W. B. Atwood, L. Baldini, J. Ballet, G. Barbiellini, D. Bastieri, K. Bechtol, A. Belfiore, R. Bellazzini, E. Bernieri, E. Bissaldi, E. D. Bloom, E. Bonamente, T. J. Brandt, J. Bregeon, M. Brigida, P. Bruel, R. Buehler, T. H. Burnett, S. Buson, G. A. Caliandro, R. A. Cameron, R. Campana, P. A. Caraveo, J. M. Casandjian, E. Cavazzuti, C. Cecchi, E. Charles, R. C. G. Chaves, A. Chekhtman, C. C. Cheung, J. Chiang, G. Chiaro, S. Ciprini, R. Claus, J. Cohen-Tanugi, L. R. Cominsky, J. Conrad, S. Cutini, F. D’Ammando, A. de Angelis, F. de Palma, C. D. Dermer, R. Desiante, S. W. Digel, L. Di Venere, P. S. Drell, A. Drlica-Wagner, C. Favuzzi, S. J. Fegan, E. C. Ferrara, W. B. Focke, P. Fortin, A. Franckowiak, S. Funk, P. Fusco, F. Gargano, D. Gasparrini, N. Gehrels, S. Germani, N. Giglietto, P. Giommi, F. Giordano, M. Giroletti, G. Godfrey, G. A. Gomez-Vargas, I. A. Grenier, S. Guiriec, D. Hadasch, Y. Hanabata, A. K. Harding, M. Hayashida, E. Hays, J. Hewitt, A. B. Hill, D. Horan, R. E. Hughes, T. Jogler, G. Jóhannesson, A. S. Johnson, T. J. Johnson, W. N. Johnson, T. Kamae, J. Kataoka, T. Kawano, J. Knödlseder, M. Kuss, J. Lande, S. Larsson, L. Latronico, M. Lemoine-Goumard, F. Longo, F. Loparco, B. Lott, M. N. Lovellette, P. Lubrano, E. Massaro, M. Mayer, M. N. Mazziotta, J. E. McEnery, J. Mehault, P. F. Michelson, T. Mizuno, A. A. Moiseev, M. E. Monzani, A. Morselli, I. V. Moskalenko, S. Murgia, R. Nemmen, E. Nuss, T. Ohsugi, A. Okumura, M. Orienti, J. F. Ormes, D. Paneque, J. S. Perkins, M. Pesce-Rollins, F. Piron, G. Pivato, T. A. Porter, S. Rainò, M. Razzano, A. Reimer, O. Reimer, T. Reposeur, S. Ritz, R. W. Romani, M. Roth, P. M. Saz Parkinson, A. Schulz, C. Sgrò, E. J. Siskind, D. A. Smith, G. Spandre, P. Spinelli, Ł. Stawarz, A. W. Strong, D. J. Suson, H. Takahashi, J. G. Thayer, J. B. Thayer, D. J. Thompson, L. Tibaldo, M. Tinivella, D. F. Torres, G. Tosti, E. Troja, Y. Uchiyama, T. L. Usher, J. Vandenbroucke, V. Vasileiou, G. Vianello, V. Vitale, M. Werner, B. L. Winer, K. S. Wood, and M. Wood, The First Fermi-LAT Catalog of Sources above $10 \mathrm{GeV}$, ApJS 209 (Dec., 2013) 34.

[3] W. B. Atwood, L. Baldini, J. Bregeon, P. Bruel, A. Chekhtman, J. Cohen-Tanugi, A. Drlica-Wagner, J. Granot, F. Longo, N. Omodei, M. Pesce-Rollins, S. Razzaque, L. S. Rochester, C. Sgrò, M. Tinivella, T. L. Usher, and S. Zimmer, New Fermi-LAT Event Reconstruction Reveals More High-energy Gamma Rays from Gamma-Ray Bursts, ApJ 774 (Sept., 2013) 76, [arXiv:1307.3037].

[4] M. Su, T. R. Slatyer, and D. P. Finkbeiner, Giant Gamma-ray Bubbles from Fermi-LAT: Active Galactic Nucleus Activity or Bipolar Galactic Wind?, ApJ 724 (Dec., 2010) 1044-1082, [arXiv:1005.5480].

[5] M. Ackermann, A. Albert, W. B. Atwood, L. Baldini, J. Ballet, G. Barbiellini, D. Bastieri, R. Bellazzini, E. Bissaldi, R. D. Blandford, E. D. Bloom, E. Bottacini, T. J. Brandt, J. Bregeon, P. Bruel, R. Buehler, S. Buson, G. A. Caliandro, R. A. Cameron, M. Caragiulo, P. A. Caraveo, E. Cavazzuti, C. Cecchi, E. Charles, A. Chekhtman, J. Chiang, G. Chiaro, S. Ciprini, R. Claus, J. Cohen-Tanugi, J. Conrad, S. Cutini, F. D’ Ammando, A. de Angelis, F. de Palma, C. D. Dermer, S. W. Digel, L. Di Venere, E. d. C. e. Silva, P. S. Drell, C. Favuzzi, E. C. Ferrara, W. B. Focke, A. Franckowiak, Y. Fukazawa, S. Funk, P. Fusco, F. Gargano, D. Gasparrini, S. Germani, N. Giglietto, F. Giordano, M. Giroletti, G. Godfrey, 
G. A. Gomez-Vargas, I. A. Grenier, S. Guiriec, D. Hadasch, A. K. Harding, E. Hays, J. W. Hewitt, X. Hou, T. Jogler, G. Jóhannesson, A. S. Johnson, W. N. Johnson, T. Kamae, J. Kataoka, J. Knödlseder, D. Kocevski, M. Kuss, S. Larsson, L. Latronico, F. Longo, F. Loparco, M. N. Lovellette, P. Lubrano, D. Malyshev, A. Manfreda, F. Massaro, M. Mayer, M. N. Mazziotta, J. E. McEnery, P. F. Michelson, W. Mitthumsiri, T. Mizuno, M. E. Monzani, A. Morselli, I. V. Moskalenko, S. Murgia, R. Nemmen, E. Nuss, T. Ohsugi, N. Omodei, M. Orienti, E. Orlando, J. F. Ormes, D. Paneque, J. H. Panetta, J. S. Perkins, M. Pesce-Rollins, V. Petrosian, F. Piron, G. Pivato, S. Rainò, R. Rando, M. Razzano, S. Razzaque, A. Reimer, O. Reimer, M. Sánchez-Conde, M. Schaal, A. Schulz, C. Sgrò, E. J. Siskind, G. Spandre, P. Spinelli, Ł. Stawarz, A. W. Strong, D. J. Suson, M. Tahara, H. Takahashi, J. B. Thayer, L. Tibaldo, M. Tinivella, D. F. Torres, G. Tosti, E. Troja, Y. Uchiyama, G. Vianello, M. Werner, B. L. Winer, K. S. Wood, M. Wood, and G. Zaharijas, The Spectrum and Morphology of the Fermi Bubbles, ApJ 793 (Sept., 2014) 64, [arXiv:1407.7905].

[6] J.-M. Casandjian, The Fermi-LAT model of interstellar emission for standard point source analysis, ArXiv:1502.07210 (Feb., 2015) [arXiv:1502.0721].

[7] R. J. Gould and G. Schréder, Opacity of the Universe to High-Energy Photons, PRL 16 (Feb., 1966) 252-254.

[8] G. G. Fazio and F. W. Stecker, Predicted High Energy Break in the Isotropic Gamma Ray Spectrum: a Test of Cosmological Origin, Nature 226 (Apr., 1970) 135-136.

[9] F. W. Stecker, O. C. de Jager, and M. H. Salamon, TeV gamma rays from 3C 279 - A possible probe of origin and intergalactic infrared radiation fields, ApJL 390 (May, 1992) L49-L52.

[10] M. Ackermann, M. Ajello, A. Allafort, P. Schady, L. Baldini, J. Ballet, G. Barbiellini, D. Bastieri, R. Bellazzini, R. D. Blandford, E. D. Bloom, A. W. Borgland, E. Bottacini, A. Bouvier, J. Bregeon, M. Brigida, P. Bruel, R. Buehler, S. Buson, S. Buson, G. A. Caliandro, R. A. Cameron, R. A. Cameron, P. A. Caraveo, E. Cavazzuti, C. Cecchi, E. Charles, R. C. G. Chaves, A. Chekhtman, C. C. Cheung, J. Chiang, G. Chiaro, S. Ciprini, R. Claus, J. Cohen-Tanugi, J. Conrad, S. Cutini, F. D’Ammando, F. de Palma, C. D. Dermer, S. W. Digel, E. S. E. Do Couto, A. Dominguez, P. S. Drell, A. Drlica-Wagner, C. Favuzzi, S. J. Fegan, W. B. Focke, A. Franckowiak, Y. Fukazawa, S. Funk, P. Fusco, F. Gargano, D. Gasparrini, N. Gehrels, S. Germani, N. Giglietto, F. Giordano, M. Giroletti, T. Glanzman, G. Godfrey, I. A. Grenier, J. E. Grove, S. Guiriec, M. Gustafsson, D. Hadasch, M. Hayashida, E. Hays, M. S. Jackson, T. Jogler, J. Kataoka, J. Knodiseder, M. Kuss, J. Lande, S. Larsson, L. Latronico, F. Longo, F. Loparco, M. N. Lovellette, P. Lubrano, M. N. Mazziotta, J. E. McEnery, J. Mehault, P. F. Michelson, T. Mizuno, C. Monte, M. E. Monzani, A. Morselli, I. V. Moskalenko, S. Murgia, A. Tramacere, E. Nuss, J. Greiner, M. Ohno, T. Ohsugi, N. Omodei, M. Orienti, E. Orlando, J. F. Omes, D. Paneque, J. S. Perkins, M. Pesce-Rollins, F. Piron, G. Pivato, T. A. Porter, S. Raino, R. Rando, M. Razzano, S. Razzaque, A. Reimer, O. Reimer, L. C. Reyes, S. Ritz, A. Rau, C. Romoli, M. Roth, M. Sanchez-Conde, D. A. Sanchez, J. D. Scargle, C. Sgro, E. J. Siskind, 
G. Spandre, P. Spinelli, L. Stawarz, D. J. Suson, H. Takahashi, T. Tanaka, J. G. Thayer, D. J. Thompson, L. Tibaldo, M. Tinivella, D. F. Torres, G. Tosti, E. Troja, T. L. Usher, J. Vandenbroucke, V. Vasileiou, G. Vianello, V. Vitale, A. P. Waite, B. L. Winer, K. S. Wood, and M. Wood, The imprint of the extragalactic background light in the gamma-ray spectra of blazars., Science 338 (Nov., 2012) 1190-1192, [arXiv: 1211.1671$].$

[11] A. Abramowski, F. Acero, F. Aharonian, A. G. Akhperjanian, G. Anton, S. Balenderan, A. Balzer, A. Barnacka, Y. Becherini, J. Becker Tjus, K. Bernlöhr, E. Birsin, J. Biteau, A. Bochow, C. Boisson, J. Bolmont, P. Bordas, J. Brucker, F. Brun, P. Brun, T. Bulik, S. Carrigan, S. Casanova, M. Cerruti, P. M. Chadwick, A. Charbonnier, R. C. G. Chaves, A. Cheesebrough, G. Cologna, J. Conrad, C. Couturier, M. Dalton, M. K. Daniel, I. D. Davids, B. Degrange, C. Deil, P. deWilt, H. J. Dickinson, A. Djannati-Ataï, W. Domainko, L. O'C. Drury, G. Dubus, K. Dutson, J. Dyks, M. Dyrda, K. Egberts, P. Eger, P. Espigat, L. Fallon, C. Farnier, S. Fegan, F. Feinstein, M. V. Fernandes, D. Fernandez, A. Fiasson, G. Fontaine, A. Förster, M. Füßling, M. Gajdus, Y. A. Gallant, T. Garrigoux, H. Gast, B. Giebels, J. F. Glicenstein, B. Glück, D. Göring, M.-H. Grondin, S. Häffner, J. D. Hague, J. Hahn, D. Hampf, J. Harris, S. Heinz, G. Heinzelmann, G. Henri, G. Hermann, A. Hillert, J. A. Hinton, W. Hofmann, P. Hofverberg, M. Holler, D. Horns, A. Jacholkowska, C. Jahn, M. Jamrozy, I. Jung, M. A. Kastendieck, K. Katarzyński, U. Katz, S. Kaufmann, B. Khélifi, D. Klochkov, W. Kluźniak, T. Kneiske, N. Komin, K. Kosack, R. Kossakowski, F. Krayzel, H. Laffon, G. Lamanna, J.-P. Lenain, D. Lennarz, T. Lohse, A. Lopatin, C.-C. Lu, V. Marandon, A. Marcowith, J. Masbou, G. Maurin, N. Maxted, M. Mayer, T. J. L. McComb, M. C. Medina, J. Méhault, U. Menzler, R. Moderski, M. Mohamed, E. Moulin, C. L. Naumann, M. Naumann-Godo, M. de Naurois, D. Nedbal, N. Nguyen, J. Niemiec, S. J. Nolan, S. Ohm, E. de Oña Wilhelmi, B. Opitz, M. Ostrowski, I. Oya, M. Panter, D. Parsons, M. Paz Arribas, N. W. Pekeur, G. Pelletier, J. Perez, P.-O. Petrucci, B. Peyaud, S. Pita, G. Pühlhofer, M. Punch, A. Quirrenbach, M. Raue, A. Reimer, O. Reimer, M. Renaud, R. de los Reyes, F. Rieger, J. Ripken, L. Rob, S. Rosier-Lees, G. Rowell, B. Rudak, C. B. Rulten, V. Sahakian, D. A. Sanchez, A. Santangelo, R. Schlickeiser, A. Schulz, U. Schwanke, S. Schwarzburg, S. Schwemmer, F. Sheidaei, J. L. Skilton, H. Sol, G. Spengler, Ł. Stawarz, R. Steenkamp, C. Stegmann, F. Stinzing, K. Stycz, I. Sushch, A. Szostek, J.-P. Tavernet, R. Terrier, M. Tluczykont, K. Valerius, C. van Eldik, G. Vasileiadis, C. Venter, A. Viana, P. Vincent, H. J. Völk, F. Volpe, S. Vorobiov, M. Vorster, S. J. Wagner, M. Ward, R. White, A. Wierzcholska, D. Wouters, M. Zacharias, A. Zajczyk, A. A. Zdziarski, A. Zech, and H.-S. Zechlin, Measurement of the extragalactic background light imprint on the spectra of the brightest blazars observed with H.E.S.S, ArXiv:1212.3409 (Dec., 2012) [arXiv:1212.3409].

[12] A. Domínguez, J. D. Finke, F. Prada, J. R. Primack, F. S. Kitaura, B. Siana, and D. Paneque, Detection of the Cosmic $\gamma$-Ray Horizon from Multiwavelength Observations of Blazars, ApJ 770 (June, 2013) 77, [arXiv:1305.2162].

[13] M. Ackermann, M. Ajello, A. Albert, W. B. Atwood, L. Baldini, J. Ballet, G. Barbiellini, D. Bastieri, K. Bechtol, R. Bellazzini, E. Bissaldi, R. D. Blandford, E. D. Bloom, 
E. Bottacini, T. J. Brandt, J. Bregeon, P. Bruel, R. Buehler, S. Buson, G. A. Caliandro, R. A. Cameron, M. Caragiulo, P. A. Caraveo, E. Cavazzuti, C. Cecchi, E. Charles, A. Chekhtman, J. Chiang, G. Chiaro, S. Ciprini, R. Claus, J. Cohen-Tanugi, J. Conrad, A. Cuoco, S. Cutini, F. D'Ammando, A. de Angelis, F. de Palma, C. D. Dermer, S. W. Digel, E. d. C. e. Silva, P. S. Drell, C. Favuzzi, E. C. Ferrara, W. B. Focke, A. Franckowiak, Y. Fukazawa, S. Funk, P. Fusco, F. Gargano, D. Gasparrini, S. Germani, N. Giglietto, P. Giommi, F. Giordano, M. Giroletti, G. Godfrey, G. A. Gomez-Vargas, I. A. Grenier, S. Guiriec, M. Gustafsson, D. Hadasch, K. Hayashi, E. Hays, J. W. Hewitt, P. Ippoliti, T. Jogler, G. Jóhannesson, A. S. Johnson, W. N. Johnson, T. Kamae, J. Kataoka, J. Knödlseder, M. Kuss, S. Larsson, L. Latronico, J. Li, L. Li, F. Longo, F. Loparco, B. Lott, M. N. Lovellette, P. Lubrano, G. M. Madejski, A. Manfreda, F. Massaro, M. Mayer, M. N. Mazziotta, J. E. McEnery, P. F. Michelson, W. Mitthumsiri, T. Mizuno, A. A. Moiseev, M. E. Monzani, A. Morselli, I. V. Moskalenko, S. Murgia, R. Nemmen, E. Nuss, T. Ohsugi, N. Omodei, E. Orlando, J. F. Ormes, D. Paneque, J. H. Panetta, J. S. Perkins, M. Pesce-Rollins, F. Piron, G. Pivato, T. A. Porter, S. Rainò, R. Rando, M. Razzano, S. Razzaque, A. Reimer, O. Reimer, T. Reposeur, S. Ritz, R. W. Romani, M. Sánchez-Conde, M. Schaal, A. Schulz, C. Sgrò, E. J. Siskind, G. Spandre, P. Spinelli, A. W. Strong, D. J. Suson, H. Takahashi, J. G. Thayer, J. B. Thayer, L. Tibaldo, M. Tinivella, D. F. Torres, G. Tosti, E. Troja, Y. Uchiyama, G. Vianello, M. Werner, B. L. Winer, K. S. Wood, M. Wood, G. Zaharijas, and S. Zimmer, The spectrum of isotropic diffuse gamma-ray emission between $100 \mathrm{MeV}$ and $820 \mathrm{GeV}$, arXiv:1410.3696 (Oct., 2014) [arXiv:1410.3696].

[14] M. Ajello, D. Gasparrini, M. Sánchez-Conde, G. Zaharijas, M. Gustafsson, J. Cohen-Tanugi, C. D. Dermer, Y. Inoue, D. Hartmann, M. Ackermann, K. Bechtol, A. Franckowiak, A. Reimer, R. W. Romani, and A. W. Strong, The Origin of the Extragalactic Gamma-Ray Background and Implications for Dark Matter Annihilation, ApJL 800 (Feb., 2015) L27, [arXiv:1501.0530].

[15] G. Dubus, J. L. Contreras, S. Funk, Y. Gallant, T. Hassan, J. Hinton, Y. Inoue, J. Knödlseder, P. Martin, N. Mirabal, M. de Naurois, M. Renaud, and CTA Consortium, Surveys with the Cherenkov Telescope Array, Astroparticle Physics 43 (Mar., 2013) 317-330, [arXiv:1208.5686].

[16] S. Carrigan, F. Brun, R. C. G. Chaves, C. Deil, A. Donath, H. Gast, V. Marandon, M. Renaud, and for the H. E. S. S. collaboration, The H.E.S.S. Galactic Plane Survey - maps, source catalog and source population, ArXiv:1307.4690 (July, 2013) [arXiv:1307.4690].

[17] S. Westerhoff, HAWC: A next-generation all-sky gamma-ray telescope, Advances in Space Research 53 (May, 2014) 1492-1498. 